\title{
Effect of Implantoplasty on Fatigue Performance and Surface Roughness of Narrow Diameter Dental Implants
}

\section{Octavi Camps-Font}

University of Barcelona

Jorge Toledano-Serrabona

University of Barcelona

Ana Juiz-Camps

University of Barcelona

Rui Figueiredo ( $\square$ ruibarbosa@ub.edu )

University of Barcelona

Cosme Gay-Escoda

University of Barcelona

Eduard Valmaseda-Castellón

University of Barcelona

\section{Research Article}

Keywords: Implantoplasty on Fatigue, Dental Implants, micrographs

Posted Date: December 21st, 2021

DOI: https://doi.org/10.21203/rs.3.rs-1164334/v1

License: (c) (1) This work is licensed under a Creative Commons Attribution 4.0 International License.

Read Full License 


\section{Abstract}

Implantoplasty (IP) is used in dental implants with peri-implantitis and aims to remove threads and polish rough surfaces in order to prevent bacterial colonization. As a result of this procedure, implant strength might be compromised. We tested 20 tapered screw-shaped Ti6Al4V dental implants with a simulated bone loss of $50 \%$. Ten implants underwent IP and 10 served as controls. Surface topography $\left(S_{a}, S_{z}, S_{s k}\right.$ and $S_{d r}$ ) was analyzed with a confocal optical microscope. Subsequently, cyclic loads were applied with a servo-hydraulic mechanical testing machine $\left(5 \times 10^{6}\right.$ cycles at $15 \mathrm{~Hz}$, between the maximal compression force - $529 \mathrm{~N}$ in the IP group and $735 \mathrm{~N}$ in the control group - and $10 \%$ of that force). We recorded the number of cycles until failure and the type of failure. Implant failure was analyzed by visual inspection and scanning electron microscopy.

Implantoplasty reduced the median $\mathrm{S}_{\mathrm{a}}$ from $1.76(\mathrm{IQR}=0.11)$ to $0.49(\mathrm{IQR}=0.16)$. The fatigue limits of the control and implantoplasty groups were $551 \mathrm{~N}$ and $529 \mathrm{~N}$, respectively. The scanning electron micrographs showed fatigue striations indicating fatigue failure. The infinite life range of the dental implants evaluated was largely above the threshold of usual chewing forces. Implantoplasty seems to render a fairly smooth surface and has a limited impact upon fatigue resistance.

\section{Introduction}

Dental implants are a predictable long-term treatment option for the esthetic and functional rehabilitation of patients with partial or total edentulism ${ }^{1,2}$. However, different complications can arise and jeopardize the results of implant-prosthetic rehabilitation ${ }^{3}$. Peri-implant diseases (both peri-implant mucositis and peri-implantitis) are considered to be the most common long-term complications associated with dental implants. Both disorders are associated to an inflammatory reaction caused by bacterial biofilm, affecting an osseointegrated dental implant ${ }^{4}$. Peri-implantitis is characterized by inflammatory changes in the peri-implant mucosa and progressive bone loss ${ }^{5}$.

Nonsurgical treatment has been shown to offer limited efficacy in the remission of peri-implantitis ${ }^{6-9}$. A possible reason could be insufficient decontamination of the implant surface, which is exposed to bacterial colonization and is usually moderately rough. In fact, the macro-geometry of the threads and the surface roughness of the implant can further complicate decontamination in the presence of associated peri-implant bone loss ${ }^{10}$. Depending on the morphology and the extent of the defect, as well as on the location of the implant, surgical treatment can involve different approaches and implant surface decontamination techniques ${ }^{11}$. Among these techniques, implantoplasty (IP) consists of polishing and smoothing those parts of rough-surfaced implants that are outside the bone contour due to progressive marginal bone loss associated with peri-implantitis, or eventual bone resection during peri-implant surgery ${ }^{12}$. Although this technique has proved effective in clinical studies ${ }^{13,14}$, several investigations have reported that IP reduces the fracture resistance of both standard (i.e., 3.75 to $4.5 \mathrm{~mm}$ ) and narrow (i.e., $\leq 3.5 \mathrm{~mm}$ ) diameter implants ${ }^{15-18}$. However, none of these studies have determined the fatigue 
limit in an unfavorable clinical scenario (i.e., narrow implants with a diameter $\leq 3.5 \mathrm{~mm}$ and bone loss equivalent to $50 \%$ of their length). These situations are common in dental implants that require IP.

Thus, the primary objective of the present study was to determine the effect of IP upon the fatigue resistance of Ti6Al4V narrow screw-shaped dental implants, with internal connection and a moderately rough surface, in the presence of $50 \%$ bone loss. The secondary objective was to analyze the implant surface roughness after IP.

\section{Results}

All samples were treated without any deviation from the protocol.

Surface topography analyses

The surface topography results are shown in Table 1. The median $S_{a}$ (arithmetic mean height), $S_{z}$ (average maximum height), $S_{\text {sk }}$ (skewness of topography height distribution), and $S_{d r}$ (developed interfacial area ratio) values of the IP group were significantly lower than those of the control group ( $p \leq$ 0.001) (Table 1). 
Table 1

Median surface roughness.

\begin{tabular}{|llllllllll|}
\hline & $\mathbf{S}_{\mathbf{a}}(\boldsymbol{\mu m})$ & & $\mathbf{S}_{\mathbf{z}}(\boldsymbol{\mu m})$ & & $\mathbf{S}_{\text {sk }}$ & & $\mathbf{S}_{\text {dr }}(\%)$ & \\
\hline Sample & Control & IP & Control & IP & Control & IP & Control & IP \\
\hline $\mathbf{1}$ & 1.88 & 0.47 & 20.86 & 13.34 & 0.29 & -1.02 & 18.95 & 2.62 \\
\hline $\mathbf{2}$ & 1.84 & 0.49 & 19.60 & 6.20 & -0.04 & -0.46 & 18.97 & 2.57 \\
\hline $\mathbf{3}$ & 1.82 & 0.58 & 30.64 & 7.66 & 0.31 & -0.86 & 18.73 & 2.61 \\
\hline $\mathbf{4}$ & 1.78 & 0.44 & 23.84 & 7.32 & -0.03 & -0.78 & 18.93 & 2.42 \\
\hline $\mathbf{5}$ & 1.73 & 0.65 & 21.11 & 15.76 & -0.07 & -0.70 & 17.67 & 5.01 \\
\hline $\mathbf{6}$ & 1.87 & 0.50 & 33.41 & 10.47 & 0.64 & -0.99 & 20.96 & 3.45 \\
\hline $\mathbf{7}$ & 1.72 & 0.33 & 20.15 & 5.04 & -0.05 & -1.39 & 16.29 & 1.19 \\
\hline $\mathbf{8}$ & 1.71 & 0.73 & 19.21 & 8.72 & 0.05 & -0.14 & 16.69 & 3.84 \\
\hline $\mathbf{9}$ & 1.74 & 0.61 & 19.78 & 9.09 & -0.14 & -0.71 & 16.35 & 2.95 \\
\hline $\mathbf{1 0}$ & 1.72 & 0.34 & 27.92 & 6.31 & 0.05 & 0.18 & 16.89 & 2.75 \\
\hline Median & 1.76 & 0.49 & 20.98 & 8.19 & 0.01 & -0.74 & 18.20 & 2.67 \\
\hline IQR & 0.11 & 0.16 & 8.14 & 4.16 & 0.34 & 0.53 & 2.26 & 0.87 \\
\hline P-value & $<0.001 *$ & & $<0.001 *$ & & $0.001 *$ & & $<0.001 *$ & \\
\hline IQR: Interquartile range; IP: Implantoplasty & & & & & \\
* Significant association $(p<0.05)$ & & & & & & & \\
\hline
\end{tabular}

\section{Fatigue testing}

Nineteen implants underwent fatigue testing: 10 in the IP group and 9 in the control group (Table 2). Three consecutive samples subjected to IP withstood the $5 \times 10^{6}$ cycles of the initial load level without apparent damage. This was equivalent to $529 \mathrm{~N}$, which corresponds to $80 \%$ of the maximum compression force $\left(F_{\max I P}\right)$. The fatigue limit of the control group was $551 \mathrm{~N}$ (i.e., $60 \%$ of $\left.F_{\operatorname{maxc}}\right)$ (Table 2).

Considering that the distance between the nominal bone level and the center of the hemispherical load abutment was $1.3 \mathrm{~cm}$, the maximum bending moments (M) were: 
Table 2

Results of the cyclic tests.

\begin{tabular}{|c|c|c|c|c|}
\hline \multirow{2}{*}{$\% F_{\max }$ total } & \multirow{2}{*}{$\begin{array}{l}\text { Peak load } \\
\text { (N) }\end{array}$} & \multirow{2}{*}{$\begin{array}{l}\text { Number of } \\
\text { cycles }\end{array}$} & \multicolumn{2}{|l|}{ Failure } \\
\hline & & & Location & Description \\
\hline \multicolumn{5}{|c|}{ Implantoplasty group $(n=10)$} \\
\hline $95 \%$ & 628 & $5,000,000$ & \multicolumn{2}{|c|}{ Absence of failure } \\
\hline $95 \%$ & 628 & $5,000,000$ & \multicolumn{2}{|c|}{ Absence of failure } \\
\hline $95 \%$ & 628 & 102,360 & Implant body & Fracture \\
\hline $90 \%$ & 595 & 279,251 & Implant body & Fracture \\
\hline $90 \%$ & 595 & $5,000,000$ & \multicolumn{2}{|c|}{ Absence of failure } \\
\hline $85 \%$ & 562 & 318,799 & Implant body & Fracture \\
\hline $85 \%$ & 562 & $5,000,000$ & \multicolumn{2}{|c|}{ Absence of failure } \\
\hline $80 \%$ & 529 & $5,000,000$ & \multicolumn{2}{|c|}{ Absence of failure } \\
\hline $80 \%$ & 529 & $5,000,000$ & \multicolumn{2}{|c|}{ Absence of failure } \\
\hline $80 \%$ & 529 & $5,000,000$ & \multicolumn{2}{|c|}{ Absence of failure } \\
\hline \multicolumn{5}{|c|}{ Control group $(n=9)$} \\
\hline $80 \%$ & 735 & 36,364 & Implant body & Fracture \\
\hline $80 \%$ & 735 & 66,690 & Implant body & Fracture \\
\hline $70 \%$ & 643 & 38,830 & Implant body & Fracture \\
\hline $70 \%$ & 643 & 68,519 & Implant body & Fracture \\
\hline $65 \%$ & 597 & 112,841 & Implant body & Fracture \\
\hline $65 \%$ & 597 & 85,644 & Implant body & Fracture \\
\hline $60 \%$ & 551 & $5,000,000$ & \multicolumn{2}{|c|}{ Absence of failure } \\
\hline $60 \%$ & 551 & $5,000,000$ & \multicolumn{2}{|c|}{ Absence of failure } \\
\hline $60 \%$ & 551 & $5,000,000$ & \multicolumn{2}{|c|}{ Absence of failure } \\
\hline
\end{tabular}

Considering that the distance between the nominal bone level and the center of the hemispherical load abutment was $1.3 \mathrm{~cm}$, the maximum bending moments $(\mathrm{M})$ were: 
(1) $M_{I P}=1.3 \mathrm{~cm} \cdot \sin 30^{\circ} \cdot 529 \mathrm{~N}=343.85 \mathrm{~N} \cdot \mathrm{cm}$

(2) $M_{C}=1.3 \mathrm{~cm} \cdot \sin 30^{\circ} \cdot 551 \mathrm{~N}=358.15 \mathrm{~N} \cdot \mathrm{cm}$

Load versus the number of cycles (S-N curves) in the IP and control groups is represented in Figure 1. Two different regions could be identified: 1 ) the finite life region was found above $551 \mathrm{~N}$ (i.e., $60 \%$ of $\left.F_{\max }\right)$; and 2 ) the infinite life range which started below that threshold. Similarly, in the load versus the number of cycles plot obtained for the IP samples (Figure 1), we determined: 1) a transition region above $529 \mathrm{~N}$ (i.e., $80 \%$ of $\mathrm{F}_{\max }$ ) ; and 2 ) an infinite life range that started below that threshold.

All failed samples exhibited a fracture pattern perpendicular to the longitudinal axis of the implant in a region of the implant body close to the embedding plane (Table 2). This area is the least thickest zone, due to the presence of the hollow space for the prosthetic screw.

\section{Fractographic analysis}

The micrographic analysis of the fracture surface revealed a typical brittle intergranular fracture mechanism. In all cases, fatigue failures started at the implant body, with subsequent fracture of the prosthetic screw. More specifically, the fracture began on the side of the implant subjected to continuous and oscillating stresses. Accumulated damage led to rupture on exceeding the mechanical resistance of the material (Figure 2).

\section{Discussion}

The present in vitro study assessed the reduction of fatigue strength of narrow-diameter dental implants with internal hexagonal connection in a model that simulated a horizontal peri-implant defect equivalent to $50 \%$ of the implant length. To the best of our knowledge, this is the first study to analyze the effect of IP in this worst-case scenario. Although implantoplasty significantly reduced the fatigue limit, the failure threshold was still above the usual chewing forces ${ }^{19,20}$. In addition, changes in surface roughness were evaluated by confocal microscopy. Implantoplasty significantly reduced the roughness of the implant surface to a minimally rough, groove-free surface - the resulting roughness being comparable to that of a machined implant surface.

In our study, IP was carried out under conditions that simulated the real-life clinical scenario, though less challenging. As previously reported with a similar protocol ${ }^{21}$, roughness zones might be difficult to reach in clinical practice, particularly in areas with difficult access or when it is not possible to remove the prosthesis. This might result in more aggressive thinning of the implant walls and, consequently, poorer mechanical properties than those reported herein.

One of the major concerns related to IP is the mechanical behavior of the dental implant after polishing 22. Although Schwarz et al. ${ }^{23}$ recommended that IP should be limited to the threads of the implant, a reduction of the thickness of the implant walls is to be expected ${ }^{18,21,24-28}$. Aside from the dimensions of 
the implant walls, other factors influence the mechanical behavior of dental implants: the implant material ${ }^{29}$, the implant-abutment connection design ${ }^{30,31}$, implant diameter ${ }^{15,18,32,33}$, crown to implant ratio ${ }^{25}$, crown height ${ }^{34}$, and nominal bone level ${ }^{26}$. This is the reason why we selected a worst-case scenario involving commercially pure titanium narrow diameter $(<3.5 \mathrm{~mm})$ implants, with an internal connection and thin walls, an unfavorable crown-to-implant ratio, and with a significant loss of supporting bone.

In a previous study ${ }^{21}$ we performed compression tests and found IP to significantly reduce fracture resistance $(p<0.001)$. Specifically, the external hexagonal, internal hexagonal and internal conical connection groups exhibited a decrease in $\mathrm{F}_{\max }$ of $27.96 \%, 28.00 \%$ and $29.41 \%$, respectively ${ }^{21}$. However, the clinical relevance of these static loading tests is limited, because factors such as time or environment are not taken into account ${ }^{35}$. In fact, mechanical failure of dental materials usually occurs once they withstand ${ }^{36}$ repeated cycles of low-energy stress, rather than higher static loads. Three in vitro studies have analyzed the effect of cyclic loading upon the fracture resistance of implant materials ${ }^{18,27,37}$. However, to the best of our knowledge, the present study is the first to determine the effect of IP upon the dental implant fatigue limit. The application of $>1 \times 10^{6}$ cyclic loads reduces fracture strength by introducing a "mechanical aging" effect in the tested components ${ }^{27,37}$. Our results suggest that even in this worst-case scenario (i.e., $3.5 \mathrm{~mm}$ diameter internal hexagonal connection implant, with bone loss equivalent to $50 \%$ of its length and subjected to IP), implants showed an infinite life range above $500 \mathrm{~N}$, which is well above the threshold of the forces recorded during chewing and swallowing (around $250 \mathrm{~N}$ ) 19,20 .

Several IP protocols have been described in the literature. Most publications agree that IP, regardless of the burs used, significantly reduces roughness ${ }^{38}$. This could have an impact upon peri-implant health, as the composition and development of biofilms on the surface of dental materials correlate with their surface roughness and free surface energy ${ }^{39}$. Other physicochemical properties, such as surface charge or substrate stiffness, appear to be of lesser importance ${ }^{40,41}$. Regarding the surface roughness of the dental implant, $\mathrm{R}_{\mathrm{a}}$ and $\mathrm{S}_{\mathrm{a}}$ are the most appropriate parameters for predicting susceptibility to bacterial adhesion ${ }^{42}$. In fact, roughness has no influence upon bacterial adhesion at $R_{a}<0.2 \mu m^{43,44}$. Although our IP protocol resulted in a minimally rough surface $\left(\mathrm{S}_{\mathrm{a}}=0.49 \mu \mathrm{m}\right)$, this still might not be smooth enough to impede bacterial adhesion. Thus, even if complete decontamination of the surface is achieved with IP, bacterial recolonization will occur within a short period of time. Therefore, it is crucial for the prosthesis to facilitate hygiene of the treated area and, at the same time, for the patient to maintain good plaque control. Furthermore, the patient should follow a maintenance program with follow-up visits at least every 4-6 months in order to avoid reinfection or the recurrence of peri-implantitis ${ }^{45}$.

\section{Conclusions}


The infinite life range of the evaluated dental implants was largely above the threshold of usual chewing forces, with the fatigue limit of the implantoplasty group being $529 \mathrm{~N}$. Thus, implantoplasty does not seem to significantly reduce fatigue resistance even in unfavorable situations where narrow-diameter internal hexagonal connection implants are involved. With carbide burs and silicon carbide polishers, $\mathrm{S}_{\mathrm{a}}$ values $<0.5 \mu \mathrm{m}$ can be obtained. Further studies are required to determine whether these results are achievable in the real-life clinical setting.

\section{Methods}

Twenty tapered, screw shaped Ti6Al4V (titanium grade 5) commercial dental implants were tested in the present in vitro study (Biomimetic Ocean ${ }^{\circledR} 3.5 \mathrm{~mm}$ wide and $10 \mathrm{~mm}$ long with internal hexagonal connection, Avinent ${ }^{\circledR}$ Implant System, Santpedor, Spain). The surface was moderately rough after a sandblasting, acid-etching and anodization process. We used a computer-generated random sequence to allocate 10 implants per group, and subsequently performed implantoplasty of the implants in the IP group.

Implantoplasty procedure

Implantoplasty was performed following the simplified three-bur protocol described by Costa-Berenguer et al. ${ }^{24}$. We inserted a cover screw to protect the implant connection from titanium debris, and removed the threads of the coronal half of the implants using an oval-shaped tungsten carbide bur (H379.314.023 Komet, GmbH \& Co. KG, Lemgo, Germany) with an air-driven high-speed handpiece under water irrigation. Then, we polished the resulting surface with two silicon carbide polishers and the same handpiece (9618.314.030 and 9608.314.030 Komet, GmbH \& Co. KG, Lemgo, Germany) (Figure 3).

Implantoplasty was performed by an experienced clinician (O.C-F.) under loupe magnification until the 5$\mathrm{mm}$ coronal portion of the implant exhibited a uniform smooth and shiny surface. The pressure applied and the number of strokes were not standardized in order to increase the external validity of the study. A new set of burs or tips was used for every other implant. After IP, the implants were thoroughly cleaned by irrigation using distilled water and dried with compressed air. Finally, the cover screw was removed. Surface topography analyses

All samples were analyzed with a confocal optical microscope (Leica ${ }^{\circledR}$ DCM 3D, Leica Microsystems AG, Wetzlar, Germany) under 20x magnification. We determined three regions of interest of $600 \times 450 \mu \mathrm{m}$ : immediately below the smooth surface of the platform $\left(T_{0}\right)$, at $2.5 \mathrm{~mm}\left(T_{2.5}\right)$, and at $5 \mathrm{~mm}\left(\mathrm{~T}_{5}\right)$ from the platform in the apical direction (Figure 4). The images were processed using the LeicaMap ${ }^{\circledR}$ application (Leica Microsystems AG, Wetzlar, Germany).

The surface texture of each area was defined using the following normalized three-dimensional parameters: $S_{a}$ (arithmetic mean height), $S_{z}$ (average maximum height), $S_{\text {sk }}$ (skewness of topography height distribution) and $S_{d r}$ (developed interfacial area ratio). Form was previously removed, and a 
Gaussian filter of $30 \mu \mathrm{m}$ was applied for roughness and waviness. Only roughness parameters were assessed.

Cast preparation

In a second step, we embedded the implants in the same position using resin casts, in such a way that 5 $\mathrm{mm}$ of rough surface was exposed. This approach was chosen to simulate a horizontal bone resorption of $5 \mathrm{~mm}$ ( $50 \%$ of the total implant length), which is $2 \mathrm{~mm}$ more than the International Standardization Organization (ISO) 14801:2016 specifications. The epoxy resin was EA 3471 A and B Loctite ${ }^{8}$ (Henkel AG \& Company, Düsseldorf, Germany) to simulate bone (Young's modulus of elasticity $\geq 3 \mathrm{GPa}$ ).

Fatigue testing

We carried out fatigue testing in room air and at room temperature using a servo-hydraulic mechanical testing machine (MTS Bionix 370, MTS ${ }^{\circledR}$, Eden Prairie, USA) equipped with a $15 \mathrm{kN}$ load cell (MTS Load Cell $661.19 \mathrm{H}-03, \mathrm{MTS}{ }^{\circledR}$, Eden Prairie, USA). We screwed identical hemispherical abutments to each implant with the torque recommended by the manufacturer $(35 \mathrm{~N} \cdot \mathrm{cm})$. The loading center was located 13 $\mathrm{mm}$ above the resin (nominal bone level). We placed the samples in a stainless-steel clamping jaw so that loading had an angle of $30^{\circ}$ to the longitudinal axis of the implant (Figure 5).

Each specimen received a maximum of 5,000,000 cycles of a uniaxial load, perpendicular to the tangent of the dome of the hemispherical abutment. Loading range was between a maximal nominal value and $10 \%$ of this value $(R=0.1)$. To minimize the vibrations of the testing machine, sinusoidal load frequency was kept at $15 \mathrm{~Hz}$. We used TestStar II® software (MTS ${ }^{\circledR}$, Eden Prairie, USA) to record data in real time.

In accordance with ISO 14801:2016, tests were carried out applying a minimum of four series of loads, the first of which was equivalent to $80 \%$ of the maximum compression force $\left(F_{\operatorname{maxc}}\right.$ and $\left.F_{\operatorname{maxIP}}\right)$, which was determined in a previous study to be $735 \mathrm{~N}$ and $529 \mathrm{~N}$ for the control and IP samples, respectively ${ }^{21}$. At each load level, two samples were evaluated, considering $5 \times 10^{6}$ cycles as an infinite life criterion. If any of the samples collapsed before reaching the specified number of cycles, the procedure was started again with two new implants and under a lesser load $\left(20 \%\right.$ if $\geq 60 \% \mathrm{~F}_{\max }$ and $10 \%$ if $\left.<60 \% \mathrm{~F}_{\max }\right)$. When two consecutive samples reached $5 \times 10^{6}$ cycles without failure, an additional test was performed with a third sample. If the latter did not fail (i.e., 3 consecutive samples without apparent failure), this point was considered to be the fatigue limit beyond which the implant could withstand an infinite number of loading cycles. In case the fatigue limit was reached in less than four load series, additional levels $(1,2$ or 3$)$ were established by applying a load $5 \%$ higher than the previous one. The number of cycles and the state (i.e., intact or failed) of each tested specimen were recorded. Failure was defined as the elastic limit of the material, permanent deformation, loosening of the implant assembly, or fracture of any component.

Additionally, for the maximal supported load, the maximal bending moment $(\mathrm{M})$ was calculated using the equation (3):

(3) $M=l \cdot \sin 30^{\circ} \cdot F$ 
Where I is the distance (in $\mathrm{cm}$ ) from the center of the load hemisphere to the nominal bone level and $F$ (in $\mathrm{N})$ the maximal supported load.

The results of the fatigue tests were displayed in a load versus number of cycles plot (i.e., S-N curve or Wöhler's curve), which represents the number of load cycles of each sample (logarithmic scale) and the corresponding maximal load (linear scale).

Fractographic analysis

All failed specimens were assessed by visual inspection and scanning electron microscopy (SEM; Quanta-200, Field Electron and Ion Company, Hillsboro, USA) to describe the failure pattern.

Statistical analysis

Categorical variables were reported as absolute and relative frequencies. We explored normal distribution of scale variables (roughness parameters) with the Shapiro-Wilk's test and visual analysis of the P-P and box plots. The mean and standard deviation (SD) were calculated and, if a normal data distribution was ruled out, the median and interquartile range (IQR) were calculated. The Mann-Whitney U-test was used to compare the groups. The statistical analysis was carried out with the Stata14 statistical package (StataCorp ${ }^{\circledR}$, College Station, TX, USA) at a level of significance $p<0.05$.

\section{Declarations}

DATA AVAILABILITY: Datasets generated and analyzed in the current study are available from the corresponding author on reasonable request.

\section{ACKNOWLEDGEMENTS}

The authors would like to thank Mr. Joe Perkins for language editing of the manuscript.

The present research was conducted by the Dental and Maxillofacial Pathology and Therapeutics research group at the IDIBELL Institute (L'Hospitalet de Llobregat, Barcelona, Spain).

\section{AUTHOR CONTRIBUTIONS}

O.C-F, E.V-C. R.F., C.G-E. conceived and designed the study; C.G-E., E.V-C. supervised the study; O.C-F., A.JC. acquired and analyzed the data; O.C-F., J.T-S. performed the statistical analysis; O.C-F J.T-S. R.F., E.V-C. interpreted the data and were involved in preparing the manuscript. All authors reviewed the final manuscript, approved the submitted version and agreed both to be personally accountable for the author's own contributions and to ensure that questions related to the accuracy or integrity of any part of the work are appropriately investigated, resolved, and the resolution documented in the literature.

\section{References}


1. Pjetursson, B. E., Thoma, D., Jung, R., Zwahlen, M. \& Zembic, A. A systematic review of the survival and complication rates of implant-supported fixed dental prostheses (FDPs) after a mean observation period of at least 5 years. Clin. Oral Implants Res. 23, 22-38 (2012).

2. Salvi, G. E. \& Brägger, U. Mechanical and technical risks in implant therapy. Int. J. Oral Maxillofac. Implants 24, 69-85 (2009).

3. Jung, R. E., Zembic, A., Pjetursson, B. E., Zwahlen, M. \& Thoma, D. S. Systematic review of the survival rate and the incidence of biological, technical, and aesthetic complications of single crowns on implants reported in longitudinal studies with a mean follow-up of 5 years. Clin. Oral Implants Res. 23, 221 (2012).

4. Berglundh, T. et al. Peri-implant diseases and conditions: Consensus report of workgroup 4 of the 2017 World Workshop on the Classification of Periodontal and Peri-Implant Diseases and Conditions. J. Clin. Periodontol. 89, 313-318 (2018).

5. Schwarz, F., Derks, J., Monje, A. \& Wang, H.-L.. Peri-implantitis. J. Periodontol. 89, 267-290 (2018).

6. Persson, G. R., Roos-Jansåker, A.-M., Lindahl, C. \& Renvert, S. Microbiologic results after nonsurgical erbium-doped:yttrium, aluminum, and garnet laser or air-abrasive treatment of peri-implantitis: A randomized clinical trial. J. Periodontol. 82, 1267-1278 (2011).

7. Renvert, S., Lessem, J., Dahlén, G., Lindahl, C. \& Svensson, M. Topical minocycline microspheres versus topical chlorhexidine gel as an adjunct to mechanical debridement of incipient peri-implant infections: A randomized clinical trial. J. Clin. Periodontol. 33, 362-369 (2006).

8. Persson, G. R., Samuelsson, E., Lindahl, C. \& Renvert, S. Mechanical non-surgical treatment of periimplantitis: A single-blinded randomized longitudinal clinical study. II. Microbiological results. J. Clin. Periodontol. 37, 563-573 (2010).

9. Nart, J. et al. Non-surgical therapeutic outcomes of peri-implantitis: 12-month results. Clin. Oral Investig. 24, 675-682 (2020).

10. Renvert, S., Polyzois, I. \& Claffey, N. How do implant surface characteristics influence peri-implant disease? J. Clin. Periodontol. 38, 214-222 (2011).

11. Figuero, E., Graziani, F. F., Sanz, I., Herrera, D. \& Sanz, M. Management of peri-implant mucositis and peri-implantitis. Periodontol. 2000 66, 255-273 (2014).

12. Schwarz, F., John, G. \& Becker, J. The influence of implantoplasty on the diameter, chemical surface composition, and biocompatibility of titanium implants. Clin. Oral Investig. 21, 2355-2361 (2017). 
13. Romeo, E. et al. Therapy of peri-implantitis with resective surgery. Clin. Oral Implants Res. 16, 9-18 (2004).

14. Romeo, E., Lops, D., Chiapasco, M., Ghisolfi, M. \& Vogel, G. Therapy of peri-implantitis with resective surgery. A 3-year clinical trial on rough screw-shaped oral implants. Part II: Radiographic outcome. Clin. Oral Implants Res. 18, 179-187 (2007).

15. Chan, H.L. et al. Impact of implantoplasty on strength of the implant-abutment complex. Int. J. Oral Maxillofac. Implants 28, 1530-1535 (2013).

16. Gehrke, S., Junior, J., Dedavid, B. \& Shibli, J. Analysis of implant strength after implantoplasty in three implant-abutment connection designs: An in vitro study. Int. J. Oral Maxillofac. Implants 31, 65-70 (2016).

17. Tribst, J. P. M., Dal Piva, A. M. de O., Shibli, J. A., Borges, A. L. S. \& Tango, R. N. Influence of implantoplasty on stress distribution of exposed implants at different bone insertion levels. Braz. Oral Res. 31, 96 (2017).

18. Bertl, K., Isidor, F., von Steyern, P. V. \& Stavropoulos, A. Does implantoplasty affect the failure strength of narrow and regular diameter implants? A laboratory study. Clin. Oral Investig. 25, 2203-2211 (2021).

19. Steinebrunner, L., Wolfart, S., Ludwig, K. \& Kern, M. Implant-abutment interface design affects fatigue and fracture strength of implants. Clin. Oral Implants Res. 19, 1276-1284 (2008).

20. Richter, E. J. In vivo vertical forces on implants. Int. J. Oral Maxillofac. Implants 10, 99-108 (1995).

21. Camps-Font, O. et al. Fracture resistance after implantoplasty in three implant-abutment connection designs. Med. Oral Patol. Oral Cir. Bucal 25, 691-699 (2020).

22. Burgueño-Barris, G., Camps-Font, O., Figueiredo, R. \& Valmaseda-Castellón, E. The influence of implantoplasty on surface roughness, biofilm formation, and biocompatibility of titanium implants: A systematic review. Int. J. Oral Maxillofac. Implants 36, 111-119 (2021).

23. Schwarz, F., John, G. \& Becker, J. The influence of implantoplasty on the diameter, chemical surface composition, and biocompatibility of titanium implants. Clin. Oral Investig. 21, 2355-2361 (2017).

24. Costa-Berenguer, X. et al. Effect of implantoplasty on fracture resistance and surface roughness of standard diameter dental implants. Clin. Oral Implants Res. 29, 46-54 (2018).

25. Leitão-Almeida, B. et al. Effect of crown to implant ratio and implantoplasty on the fracture resistance of narrow dental implants with marginal bone loss: An in vitro study. BMC Oral Health 20, 329 (2020). 
26. Leitão-Almeida, B. et al. Effect of bone loss on the fracture resistance of narrow dental implants after implantoplasty. An in vitro study. Med. Oral Patol. Oral Cir. Bucal 26, 611-618 (2021).

27. Jorio, I. C., Stawarczyk, B., Attin, T., Schmidlin, P. R. \& Sahrmann, P. Reduced fracture load of dental implants after implantoplasty with different instrumentation sequences. An in vitro study. Clin. Oral Implants Res. 32, 881-892 (2021).

28. Sivolella, S. et al. Implantoplasty: Carbide burs vs diamond sonic tips. An in vitro study. Clin. Oral Implants Res. 32, 324-336 (2021).

29. W. Nicholson, J. Titanium alloys for dental implants: A review. Prosthesis 2, 100-116 (2020).

30. Gil, F. J., Herrero-Climent, M., Lázaro, P. \& Rios, J. V. Implant-abutment connections: Influence of the design on the microgap and their fatigue and fracture behavior of dental implants. J. Mater. Sci. Mater. Med. 25, 1825-1830 (2014).

31. Park, I. S. et al. Fatigue characteristics of five types of implant-abutment joint designs. Met. Mater. Int. 14, 133-138 (2008).

32. Shemtov-Yona, K., Rittel, D., Levin, L., Machtei, E. E. \& Levin, L. Effect of dental implant diameter on fatigue performance. Part II: Failure analysis. Clin. Implant Dent. Relat. Res. 16, 178-184 (2014).

33. Diéguez-Pereira, M. et al. Effect of implantoplasty on the elastic limit of dental implants of different diameters. Int. J. Implant Dent. 7, 88 (2021).

34. da Rocha Ferreira, J. J., Machado, L. F. M., Oliveira, J. M. \& Ramos, J. C. T. Effect of crown-toimplant ratio and crown height space on marginal bone stress: A finite element analysis. Int. J. Implant Dent. 7, 81 (2021).

35. Shemtov-Yona, K. \& Rittel, D. Fatigue of dental implants: Facts and fallacies. Dent. J. 4, 16 (2016).

36. Shemtov-Yona, K. \& Rittel, D. Identification of failure mechanisms in retrieved fractured dental implants. Eng. Fail. Anal. 38, 58-65 (2014).

37. Shah, S. D. Implant strength after implantoplasty. (The Ohio State University, 2019).

38. Ramel, C. F. et al. Surface roughness of dental implants and treatment time using six different implantoplasty procedures. Clin. Oral Implants Res. 27, 776-81 (2016).

39. Teughels, W., Van Assche, N., Sliepen, I. \& Quirynen, M. Effect of material characteristics and/or surface topography on biofilm development. Clin. Oral Implants Res. 17, 68-81 (2006).

40. Rzhepishevska, 0 . et al. The surface charge of anti-bacterial coatings alters motility and biofilm architecture. Biomater. Sci. 1, 589-602 (2013). 
41. Terada, A., Okuyama, K., Nishikawa, M., Tsuneda, S. \& Hosomi, M. The effect of surface charge property on Escherichia coli initial adhesion and subsequent biofilm formation. Biotechnol. Bioeng. 109, 1745-1754 (2012).

42. Schubert, A. et al. Predictability of microbial adhesion to dental materials by roughness parameters. Coatings $\mathbf{9}, 456$ (2019).

43. Bollen, C. M. et al. The influence of abutment surface roughness on plaque accumulation and periimplant mucositis. Clin. Oral Implants Res. 7, 201-211 (1996).

44. Quirynen, M., Bollen, C. M., Papaioannou, W., Van Eldere, J. \& van Steenberghe, D. The influence of titanium abutment surface roughness on plaque accumulation and gingivitis: short-term observations. Int. J. Oral Maxillofac. Implants 11, 169-78 (1996).

45. Serino, G., Turri, A. \& Lang, N. P. Maintenance therapy in patients following the surgical treatment of peri-implantitis: A 5-year follow-up study. Clin. Oral Implants Res.26, 950-956 (2015).

\section{Figures}

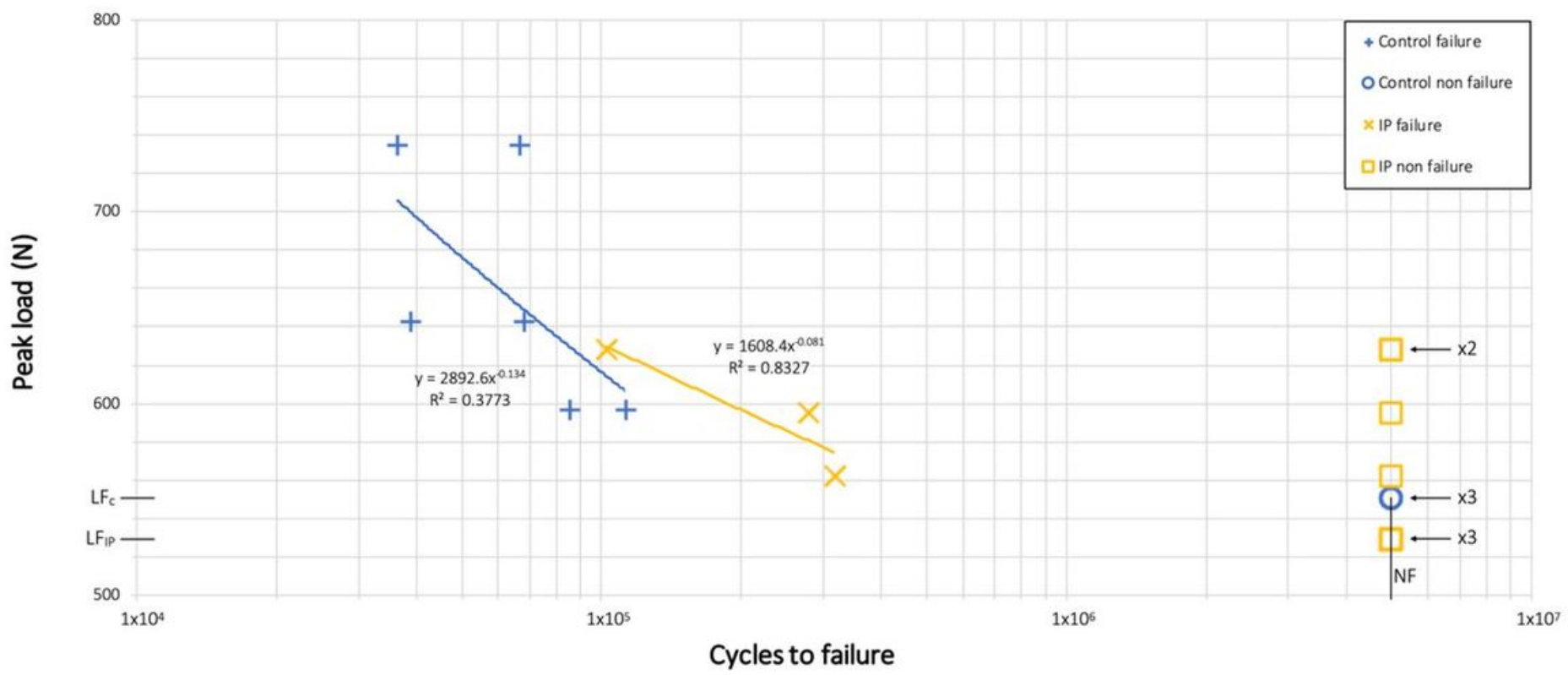

\section{Figure 1}

\section{Load versus the number of cycles plot (S-N curve) for each group.}

IP: Implantoplasty; NF: Number of load cycles (i.e., $5 \times 10^{6}$ ); LF: Fatigue limit. 

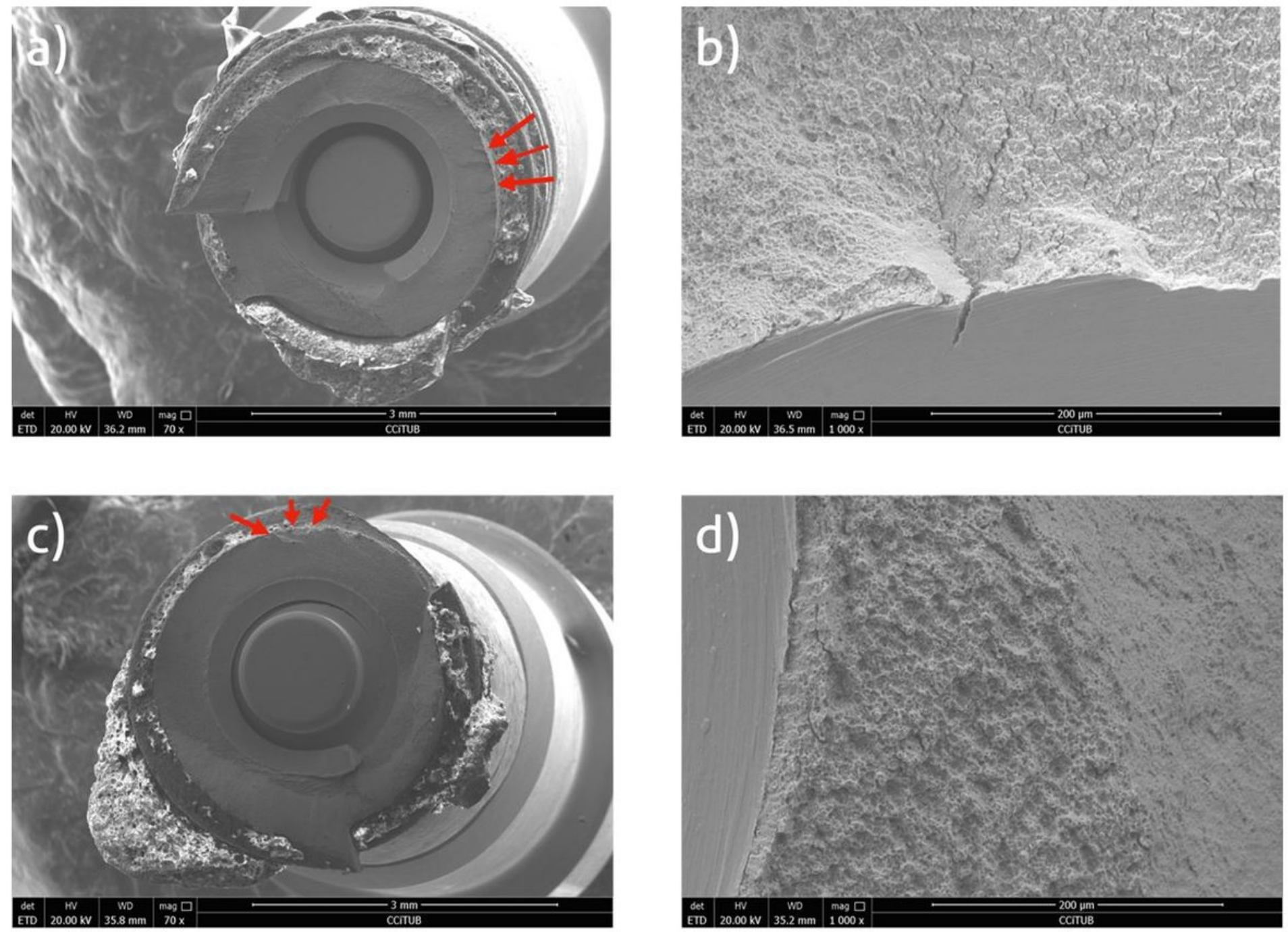

Figure 2

Fractographic analysis with scanning electron microscopy. a) Control sample at 70x and 1000x; b) Test sample at 70x and 1000x. Red arrows indicate the presence of fatigue striations. 

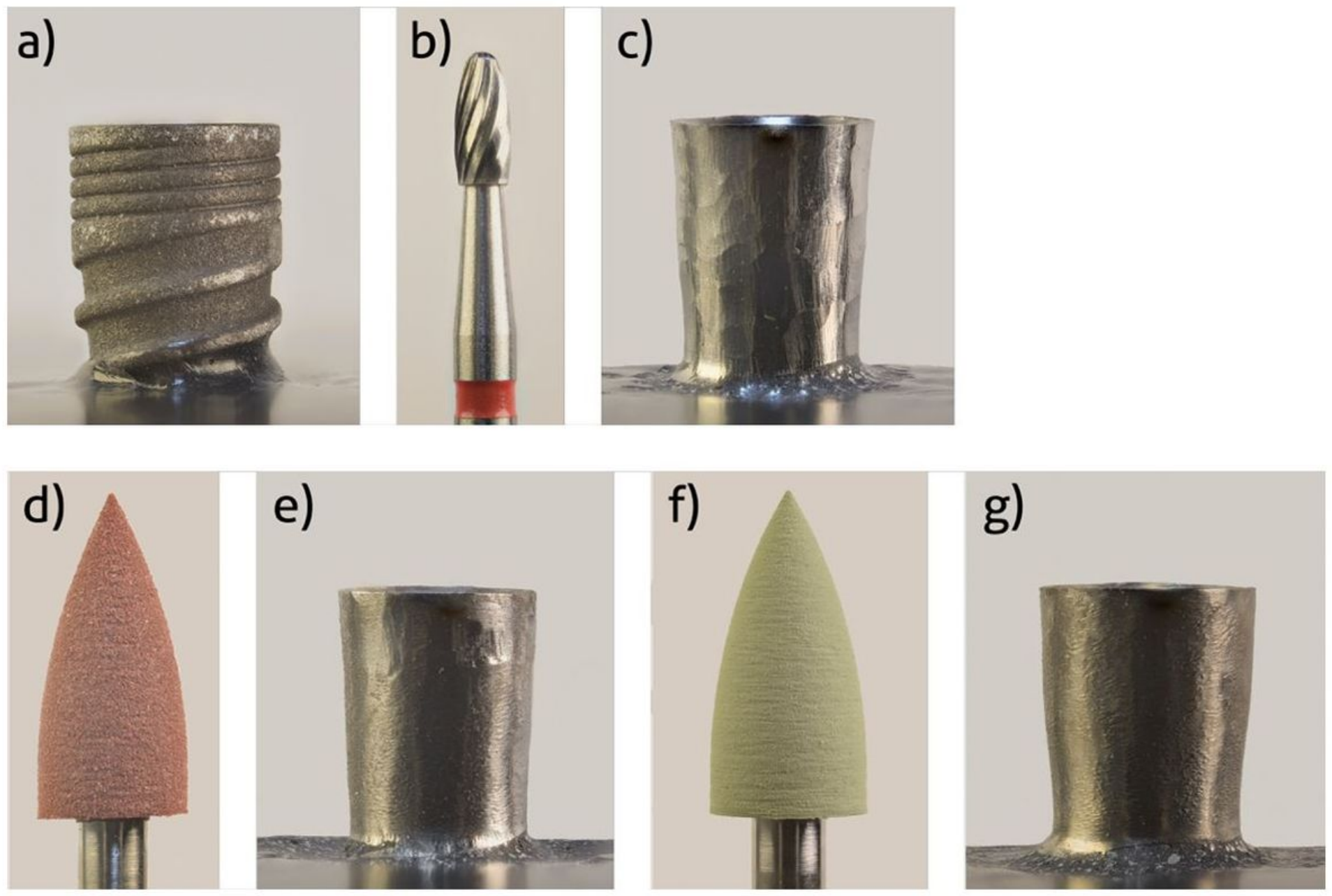

Figure 3

Simplified three-bur protocol IP procedure. a) Macroscopic appearance of the implant; $b$ and c) Macroscopic appearance of the implant after applying the tungsten carbide bur; d-e) Macroscopic appearance of the implant after applying the silicon carbide polishers.
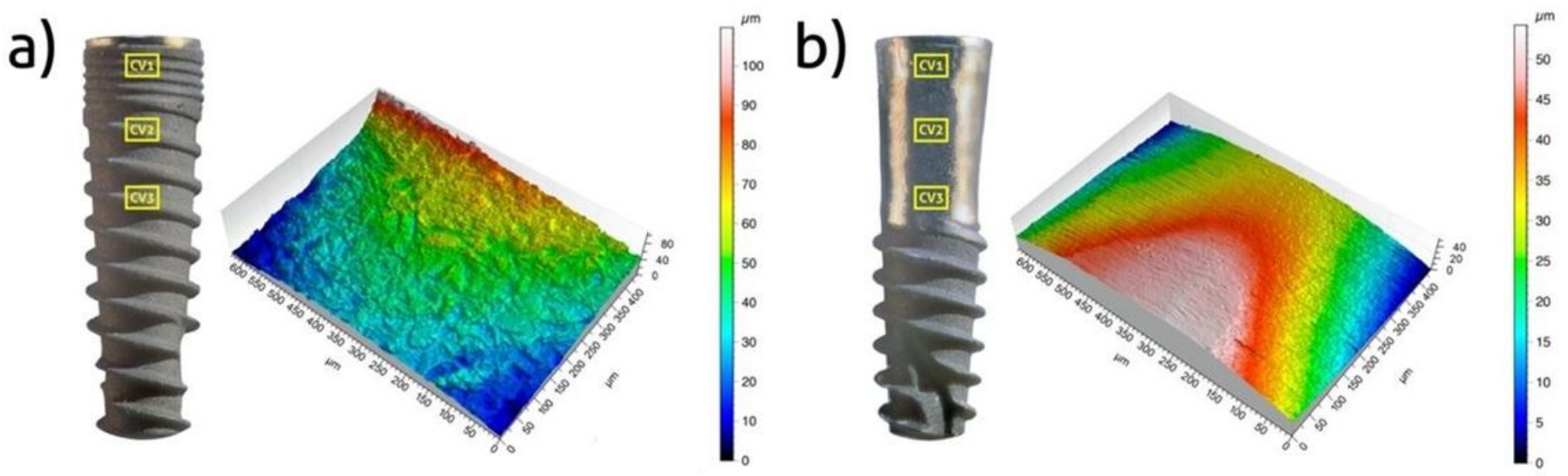

Figure 4 
Confocal microscopy surface topography analyses. a) Control group sample; b) IP sample. CV: Area of interest.

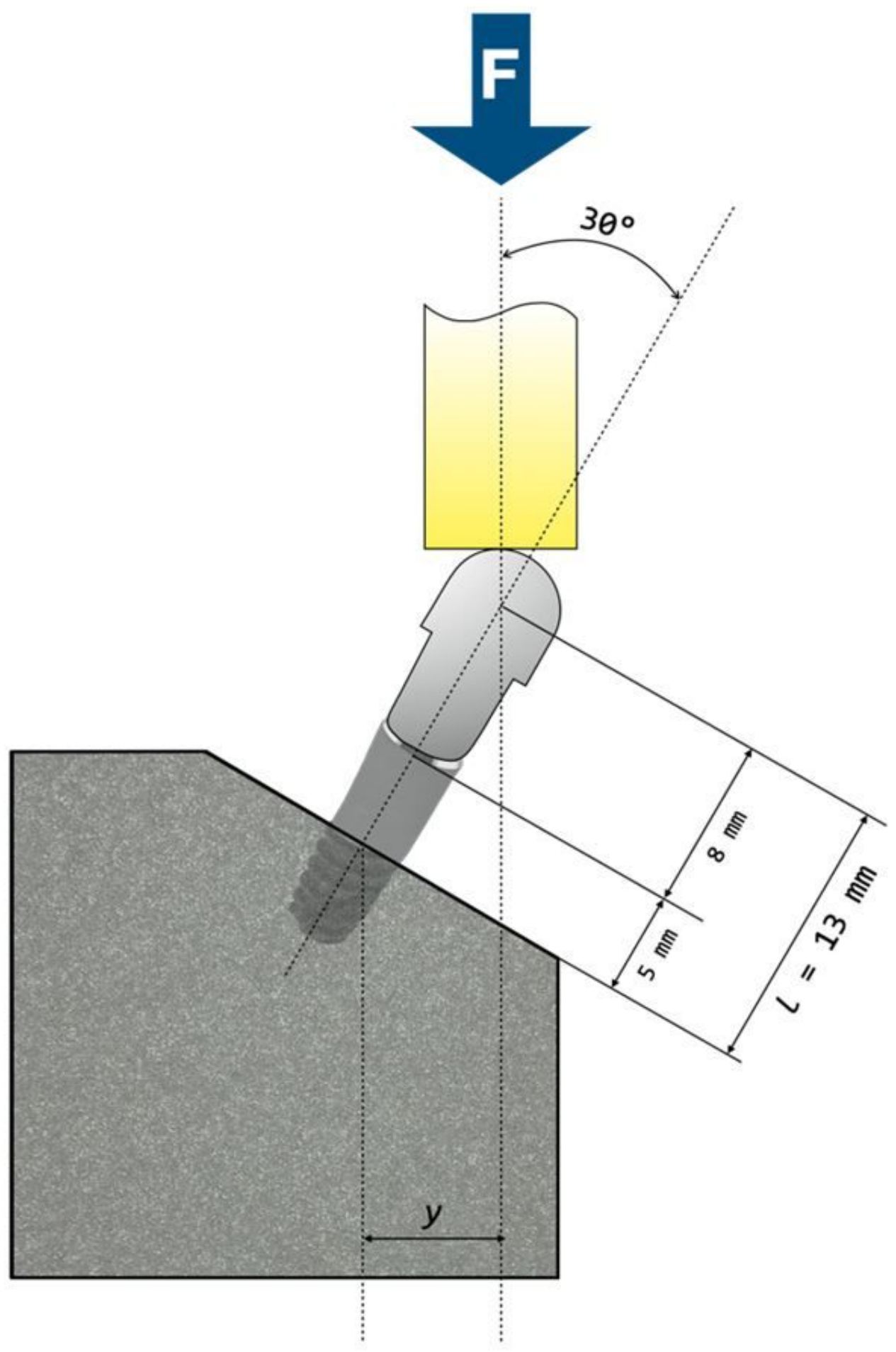

Figure 5 
Schematic representation of the test setup according to ISO 14801:2016, except for bone nominal level. 